\title{
Learning Disabilities Concept and Identification: Primary Teachers' Perspectives in Saudi Arabia
}

\author{
Fahad Alnaim
}

\begin{abstract}
Identification of Learning Disabilities LD is a significant stage in supporting pupils which requires caution, in that it might incorrectly exclude some students who have LD or include others who do not have them. In terms of the concept, the term used in this paper is 'learning disabilities' (LD) because this is the one utilised in Saudi Arabia. However, this term is not internationally used. In the UK it is called 'specific learning difficulties' (SpLD) or 'dyslexia'. In 1996, the Department of Learning Disabilities in Saudi Arabia launched the Saudi Learning Disabilities Programme (SLDP) in primary schools to identify and serve Students With Learning Disabilities (SWLD). This paper will address the concept of $L D$ and the effectiveness of the identification strategies through exploring teachers' perceptions. The discussion will be based on the pilot study which assisted to develop the data collection methods of main study.
\end{abstract}

Index Terms-Dyslexia, identification, learning disabilities, specific learning difficulties.

\section{INTRODUCTION}

The Saudi education has paid more attention to special educational needs and, particularly, learning disabilities area. In 2006, the SLDP has been established in 728 and 498 primary boys and girls schools, respectively, serving 15,038 students in total [1]. The Department of Learning Disabilities approved a manual in 2012 that names 1285 primary boys schools that have adopted the SLDP. This rapidly growing use of the SLDP in schools shows how much attention Saudi educationists pay to LD, and how significant the need for this service is perceived to be. The growing practice should be consistent with a number of pieces of research in order to ensure success, create development based on a scientific basis, take advantage of recent theories, and discover and reform underlying problems.

The researcher conducted a pilot study which includes three interviews and two focus groups with teachers of SWLD. There are important points that arose from the pilot study that should be taken into account. There are differences in identification process within reality between teachers as well as the implementing the guidance. Secondly, some teachers lack conviction in the identification procedures, especially for certain regulations which always have to be followed. For example, the limit of 8 students to programme that a teacher serves, leads teachers to always identify 8 SWLD; and, if there unfilled places in the SLDP they might select even those students who are not clearly have learning disability. However, if there too many

Manuscript received July 16, 2014; revised September 18, 2014.

The author is with the University of Manchester, Manchester, M139PL, UK (e-mail: fahad.alnaim@postgrad.manchester.ac.uk). students who are identified as having learning disability, they will be in the waiting list for next term or year. Another example is that 'observation' is highlighted as an important and required step on the identification according to the guidance [2]. However, it has been found in the pilot study that the many teachers do not undertake it, while others do it in certain cases. Interestingly, their justifications of undertaking observation are different; for example, their views about time efficiency or effectiveness of observation highlighted an important theme which influences their usage of this method.

It can be clear from this overview that there are different critical questions that need to be investigated to understand: their views about LD, how and why teachers practice identifying SWLD using different methods, and their dealing with regulations and guidance from Ministry of Education.

\section{CONCEPT AND DEFINITION}

The concept and definitions of LD should be given at the beginning to provide a greater understanding and overview, as well as emphasise the significance of the issue. Investigating various LD definitions is expected to allow the researcher to conduct deeper discussions with teachers, and allow readers to understand different views and how they can affect the way of addressing this issue. LD, as with many complex subjects, does not have one agreed definition internationally [3], [4]. This means that the different definitions might have been constructed through various perspectives and influenced by factors such as theories, practice and economics. In terms of the concept, the term used in this study is 'learning disabilities' (LD) because this is the one utilised in Saudi Arabia. However, this term is not internationally used. In the UK it is called 'specific learning difficulties' (SpLD) or 'dyslexia'. [5] and [6] indicate that SpLD include sub-categories, including dyslexia, dyspraxia, attention deficit hyperactivity disorder, and dysphasia.

The UK is a leading country in the area of LD. It was involved in deep-rooted research that studied LD more than a century ago. This consideration of LD has lasted until the present and has assisted the reform, construction and development of the understanding of various LD issues. The earliest consideration of LD in the UK was in 1896, when The British Medical Journal published an article by W. Pringle Morgan entitled "A Case of Congenital Word Blindness" [7]. The study built a basis for diagnosis of developmental dyslexia in subsequent research.

Many definitions of dyslexia have been developed since 1896. Dyslexia has been defined internationally in different ways, based on various identification, exclusionary and 
descriptive criteria. The approach to defining dyslexia will be discussed in this study in order to understand the basis of each definition and the differences between them. In what follows, two leading definitions from the UK will be presented, followed by exploratory commentaries. The British Psychological Society BPS [8] has provided a working definition of dyslexia. The BDA Management Board gave another definition in 2007.

The BPS opted for a working definition. Reference [9] suggests that this is because the BPS, as a working party, is not interested in providing causal explanation. The BPS report [8] highlights that the working definition is linked to various explanations related to delivering an initial point for producing several hypotheses linked to different theories in psychological research. While the working definition has no exclusionary criteria, the focus of positive identifying of traits was mainly on severe and persistent problems, as well as a lack of language development. These problems are more valued in the definition than other factors, such as overall ability [8], [10].

The BDA definition seems to be more comprehensive, as the definition addresses different issues in dyslexia including its forms, age, dysfunction sources and treatments. The reason behind the broadness of this definition seems to be the wide scope of the objectives and mission of the BDA. In other words, the aims of the BDA are not limited to one side of dyslexia, such as identification or teaching, as it seeks to address the subject as a whole by, for example, setting the standards for and accrediting dyslexia knowledge and professional expertise, and campaigning and lobbying for long lasting and sustainable change for the benefit of dyslexic people [11].

The above two definitions address the forms of dyslexia that can be compared with Saudi views and practice. The BDA definition highlights problems in the context of teaching methods that are similar to Saudi practice, in that class tutors are asked to identify dyslexic students who are not responding their teaching. This point in the definition (teaching methods) answers a question that might be asked as to what the purpose is behind categorising students with learning disabilities in certain forms and whether there is a solution in adopting different teaching methods. Categorising this group of learners will facilitate searching, discussion and studying of their problems, needs and treatments. By studying, effective knowledge and results can be expected, resulting in much time, effort and resources being saved by class tutors, in addition to better educational outcomes for SWLD. However, if class tutors attempt to just try different methods they will probably end up expending too much effort and achieve unsatisfactory results.

Labelling learners with $L D$ is an important issue related to the categorisation and terming. The issue is debatable and has a variety of pros and cons [12]. One of the most important issues to labelling learners with SEND is realising what this label implies, the impact it can have and how it might be understood by learners, parents and teachers [12] . Basically, the impact of the label 'dyslexic', 'learning disabled' or 'SWLD' should be reviewed using research and approaches found as to how to manage and get the most out of it. For example, [13] conducted a study at the higher education level to investigate barriers faced by students labelled as learning disabled. The results showed that barriers could be overcome through methods such as raising faculty awareness about LD issues. The research provided an example as to how labelling can be dealt with by investigating both the positive and negative impact it can have, as well as exploring solutions to reduce any possible harm.

Another country that should be considered in literature is the US, especially since the Saudi educational system is based on the US system. Therefore, the Saudi Learning Disabilities Programme (SLDP) is likely to be affected, to a greater or lesser extent, by US theories and practice in the field of LD. The SLDP is not the only programme affected by the US, as many other countries and programme around the world keep an eye on US progress in the LD field and take advantage of it. [2] claims that the early definitions of LD adopted in the US have been followed by numerous countries. He suggests that the most common definition is the one formed by the U.S. Office of Education [14].

Although that definition does not provide details about the symptoms, causes or appearance time of LD, it presents the basis of what can be included or excluded in this term (LD). Unsurprisingly, the General Secretariat for Special Education [15] in the Ministry of Education of Saudi Arabia provides a similar definition to the US one. The Saudi definition states:

'Disorders in one or more of the basic psychological processes involved in understanding or using spoken and written language which is manifested in disorders in listening, thinking, talking, reading, writing, spelling, or arithmetic and it is not due to factors related to mental retardation, visual or hearing impairments, or educational, social, and familial factors' [15].

Because of this similarity between the two definitions, other similarities and relationships between Saudi and US practice can be expected. However, the education systems and related conditions of these two countries should be understood when judging or addressing SLDP.

Having reviewed key definitions of $\mathrm{LD}$, it is important to explore significant themes of what has been discussed in relation to the current study. The definitions indicate that reading and writing difficulties are not problems in themselves but they are signs of other complicated problems. This claim is clarified in the summaries of the definitions shown in the following Table I; all of them present the phenomenon (i.e. learning difficulties) followed by an examination of the basic problems (e.g. phonological processing). The BDA definition explains that development of literacy is affected by problems such as phonological processing, working memory, and the automatic development of skills. The US and Saudi definitions highlight that many learning difficulties are manifest by disorder in one of the basic psychological processes. The BPS indicates that dyslexia is evident when there are reading or spelling difficulties. However, since the BPS provided a working definition, no further details were provided about the basic problems except that they are severe and persistent.

Since the concept of LD raises various questions, it is important to investigate how teachers of SWLD perceive this concept. In other words, when exploring a service 
provided for SWLD, such as SLDP, the exploration should take into account whether teachers of SWLD are aware of basic problems or whether they just consider any reading and writing difficulties as the core of the LD concept. The importance of their understanding of LD becomes clearer when identifying SWLD, when building a plan to address them and when serving them. For example, if teachers who are responsible for identifying SWLD think that SWLD are all those students who cannot read or write very well, the percentage of SWLD will probably be very high, as the number will include students without LD. Also, planning and serving may focus on the phenomenon even though it is crucial to construct the service with a clear understanding of the basic problems that are behind the phenomenon. In the current study, the researcher aimed to explore teachers' understanding of LD through direct and indirect questions and how this affects the identification of LD.

TABLE I: SUMMARY OF THE SELECTED DEFINITIONS

\begin{tabular}{|c|c|c|}
\hline \multicolumn{2}{c|}{ TABLE I: SUMMARY OF THE SELECTED DEFINITIONS } \\
\hline Resources & Phenomenon & Basic Problems \\
\hline BPS & $\begin{array}{c}\text { Reading or spelling } \\
\text { difficulties }\end{array}$ & ---- \\
\hline BDA & $\begin{array}{c}\text { Lack of literacy } \\
\text { development }\end{array}$ & $\begin{array}{c}\text { Phonological processing, } \\
\text { working memory, and the } \\
\text { automatic development of } \\
\text { skills }\end{array}$ \\
US and & $\begin{array}{c}\text { Many learning } \\
\text { difficulties, e.g. } \\
\text { reading, writing, } \\
\text { spelling, or } \\
\text { arithmetic }\end{array}$ & $\begin{array}{c}\text { Disorder in one of the } \\
\text { basic psychological } \\
\text { processes }\end{array}$ \\
\hline
\end{tabular}

\section{PRoblem STATEMENT}

The study aims to investigate the Saudi Learning Disabilities Programme (SLDP) in terms of the identification of Students With Learning Disabilities (SWLD). This investigation will take place by exploring the perspectives of SELD tutors. Two aspects will be included in this exploration: firstly, discovering the identification strategies which are adopted in the SLDP in reality, and secondly, examining the effectiveness of these strategies. Tutors of SWLD are the main source of the study because they are the main people responsible for the SELD, including identifying, teaching and evaluating SWLD. In addition, they are the only specialists in LD in schools.

In order to understand how the SLDP is constructed and adopted, four important factors should be considered. Firstly, policy of the SLDP seems to be affected by international views of LD. It is important while investigating the program to explore and evaluate how the SLDP is affected by these views. Secondly, some differences between policies and practitioners of the SLDP have been found in the pilot study. This shows that policies do not reflect what is being practiced in real life. In addition, a lack of details has been found while revising these policies. Thirdly, some external factors (i.e. which may caused, for instance, by commands of school administrations as a result of a lack of consideration of the SLDP) may affect aspects such as performance. Finally, beliefs and thoughts of those who play a role in the SLDP, particularly teachers of SWLD because they are in the main responsible for the SLDP. Besides the importance of exploring these views and belief, it is very important to consider how they are built up, how similar or different they are, and what influences can or usually affect them.

\section{MethodologicAl DESIGN}

There are three steps in the data collection process. The first one will be interviews to determine key issues. Next will be focus groups to get a deep understanding of the issues. Finally, a survey will find out how widely these views are held. Following paragraphs will provide more details that support to follow these methods.

Since literature does not provide a clear image about what happens practically in the SLDP, rather just the effectiveness of it, there is a need for a suitable exploration that answers this question. Appropriate data should provide enough clarification of the questions with a considerable range of deepness, broadness and credibility, based on experiences, ideas and attitudes of teachers. These features of data (deepness, broadness and credibility) are very important and influence the methods selection.

\section{A. Deepness}

Since the research questions are related to understanding the phenomenon through teachers' perspectives, it is necessary to explore their experiences, understanding and feeling about each of the related points raised. In particular, teachers probably come across many cases of SWLD and receive different training, which can be expected to affect their experiences. To be aware of how teachers construct their perspectives, it is necessary to get these teachers involved in considering their journey in terms of the issue concerned.

Significantly, interview and focus group methods are characterised by their ability to seek in-depth data, although an interview elicits more in-depth responses and gets an interpretive perspective, while a focus group allows identification of group norms and discovers various opinions within a population [16]. In-depth information can be gained from an interview through reflecting, prompting and probing techniques. An interview allows the interviewer to reflect on what has been said by the interviewee, meaning the interview provides the opportunity to reflect on the quality, adequacy and relationship of the data provided. This reflection is crucial, especially in the gap between interview [17]. Subsequently, if there is an answer given that is not clear or needs more details, the interviewer should prompt the interviewee to elucidate the response or probe the question deeper [18], [19]. Interview and focus group will be used to answer both research questions although interview seems to be the more appropriate method for the first question while a focus group seems better for the second question. The first question requires in-depth explanation about how the SLDP is applied, as well as exploration of any related points. On the other hand, the second question needs to find out how teachers reflect on the applied methods and whether they agree or disagree with other people in the focus group.

\section{B. Broadness}

Since the questions are about teachers' perspectives, it is 
necessary to collect data from a number of teachers in such a way that their quantity and diversity reflects what is typical for the study population. If the participants are few, the results might lack major points or might represent odd opinions as popular perspectives. Although interviews can involve many participants, questionnaires are more capable of increasing quantity and diversity [19]. For example, it can be difficult in Saudi culture to conduct face-to-face interviews with the opposite gender. This issue meets the researcher needs to look for other ways to generate data, such as approaching the participants using a different type of interview (e.g. telephone interview). However, far more simple is a questionnaire as it does not matter whether participants are near or far, the same or different genders; they can be approached for this study without the problems highlighted that are related to interviewing.

\section{Credibility}

It is crucial to make sure that teachers' perspectives are understood correctly. To achieve this, their perspectives should be perceived through different methods. As mentioned earlier, there are three main methods (interview, focus group and questionnaire) that need to be used. Each one of them will assist in gaining data in different forms, allowing explanation, confirmation and reflection. To achieve this, a comparison between quantitative and qualitative data will be adopted. The interpretation of numerical data will be compared with themes and patterns that emerge from verbal data.

In this progressive focus technique, eight interviews, 4-6 focus groups and 20-30 questionnaires are planned to be undertaken. the researcher will start with the interviews and then analyse what will be learnt in order to write questions for the focus groups. Similarly, the data collected from the focus groups will be analysed to write the questionnaire items. In this way, the information can be narrowed down and the investigation will be more focussed on the most significant points (see Fig. 1).

The researcher conducted a pilot study and adopted the three methods. Certain changes have been made to adapt these methods according to Saudi culture, teachers' suggestions and the researcher's observations.

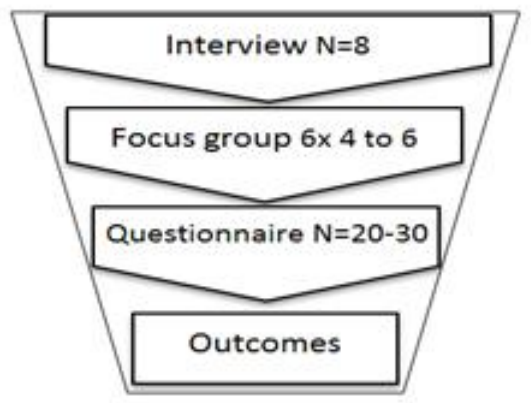

Fig. 1. Progressive focus technique.

\section{DATA ANALYSiS}

The study will follow a thematic analysis, an approach that has been addressed deeply by [20]. This approach has various advantages, such as that there is more flexibility for the researcher and the researcher does not require much experience. In relation to flexibility, the identification and analysis of themes can be done in flexible and different ways, which gives more room for the researcher to manoeuvre. Accordingly, [20] highlight that this method is "accessible to researchers with little or no experience of qualitative research". In addition, the interviews and focus groups will involve different aspects of the teachers' experience, views and perspectives, which requires identifying patterns and connecting them through links in order to help analyse and report them. The purpose of the analysis is not to examine the story given by the teacher, which could be done through narrative analysis [21], nor to explore a phenomenon by gaining rich details on how many cases people perceive, which could be done through interpretative phenomenological analysis [22]. Furthermore, the analysis is not aimed at constructing theory or analysing data based on the researcher's experience.

Thematic analysis can be done in different ways. For example, [23] provide three processes of qualitative data analysis: data reduction, data display and conclusion drawing. Also, [20] highlight six phases as qualitative analysis guidelines. The analysis in this study will follow six phases, as it seems that the six phases include the three processes in somewhat greater detail. These phases will help the researcher to be more confident in considering any important processes of his analysis.

\section{CONTRIBUtion TO KNOWLEDGE}

Internationally, the over-reliance on developed countries, western-based models and conceptualisations means that other contextual and societal idiosyncrasies and culturespecifics are often ignored, which may cause these societies difficulties and dilemmas. Therefore, there is a need to enrich the subject of LD by drawing insights from different contexts and experiences. For example, this study is expected to provide an understanding of Saudi teachers' views on LD and how they can be assisted. Thus, the nature of learning disabilities in Saudi Arabia in the teachers' views will be clearer for the world to see. Accordingly, it is important for those who are interested in $L D$ to be acquainted with learning disabilities programmes that have been successfully implemented in other societies. In doing so, a nation that is planning to introduce a learning disabilities programme can adapt certain aspects and concepts of established programmes so that they closely fit with their system of education, culture and values.

Nationally, Saudi Arabia is seeking educational development, including in the area of LD, which is why a growing number of schools are conducting the SLDP. This developing practice should be supported, evaluated and accelerated by appropriate research. In doing so, it will probably influence Saudi policy in this area. Policy makers should consider the results that will emerge from this study and rethink what goes well and does not go so well.

\section{CONCLUSION}

LD is a worldwide phenomenon, a matter that makes dispute about the issue more likely. Furthermore, the dispute 
is not limited to the definition only as it also includes the concept. Investigating into SLDP enable to presents how LD is understood by Saudi teachers. Although there is no research addressed the SLDP in terms of evaluation of effectiveness, this study is probably not the best place to start this evaluation. It is better, as the study aims, to start with the process and look the factors in this kind of process, so the implementation of it will then inform more sophisticate understanding of effectiveness.

\section{REFERENCES}

[1] Y. Alhabib, "Achievements of the Saudi Arabia Ministry of Education in the area of area of learning disabilities," presented at The international conference for learning disabilities. Riyadh, Saudi Arabia, 2006

[2] S. Almoady, Y. Bokhary, M. Alhawas, S. Almayah, and S. Alabdullatif, The Guidebook for Teachers of Learning Disabilities, Riyadh: Ministry of Education, 2013.

[3] British Dyslexia Association. (2007). Definition of Dyslexia.. [Online] Available: http://www.bdadyslexia.org.uk/about-dyslexia/furtherinformation/dyslexia-research-information-.html

[4] BPS. Dyslexia Literacy and Psychological Assessment, Leicester: British Psychological Society, 1999.

[5] V. Braun and V. Clarke, "Using thematic analysis in psychology," Qualitative Research in Psychology, vol. 3, pp. 77-101, Jan. 2006.

[6] P. Corbetta, Social Research Theory, Methods and Techniques, London: SAGE Publications, 2003.

[7] C. Dawson, A Practical Guide to Research Methods, Oxford, How To Books, 2007.

[8] H. Denhart, "Deconstructing barriers: perceptions of students labeled with learning disabilities in higher education," Journal of Learning Disabilities, vol. 41, pp. 483-97, no. 2008.

[9] U. S. Office of Education, "Assistance to states for education of handicapped children: Procedures for evaluating specific learning disabilities," edited by Federal Register, 65082-85, 1977.

[10] N. Frederickson, Norah, and U. Frith, "Identifying dyslexia in bilingual children: a phonological approach with inner London sylheti speakers," Dyslexia, vol. 4, no. 3, pp. 119-31, Sep. 1998.

[11] D. E. Gray, Doing Research in the Real World, London: SAGE, 2004

[12] A. Lieblich, R. T. Mashiach, and T. Zilbert, Narrative Research: Reading, Analysis and Interpretation, London: Sage, 1998.
[13] M. B. Miles and A. M. Huberman, Qualitative Data Analysis, 2nd ed. Thousand Oaks, CA: Sage, 1994.

[14] Ministry of Education, "The rules and law for special education schools and programs in ministry of education," Saudi Arabia: Ministry of Education, 2002.

[15] W. P. Morgan, "A case of congenital word blindness," British Medical Journal, vol. 2, p. 1378, 1896

[16] M. Patton, Qualitative Research and Evaluation Methods. 3rd ed. Thousand Oaks, CA: Sage, 2002.

[17] G. Reid, Dyslexia: A Practitioners Handbook, 4th ed. Chichester: John Wiley and Sons Ltd, 2009.

[18] B. Riddck, "Labelling learners with 'send': the good, the bad and the ugly," in Contemporary Issues in Special Educational Needs, D. Armstrong, and G. Squires eds., Maidenhead: Open University Press, pp. 25-34, 2012.

[19] G. D. Sideridis, "International approaches to learning disabilities: More alike or more different?" Learning Disabilities Research and Practice, vol. 22, pp. 210-15, 2007.

[20] J. A. Smith and M. Osborn. "Interpretative phenomenological analysis," in Qualitative Psychology: A Practical Guide to Research Methods, J. A. Smith ed., London: Sage, pp. 15-80, 2003.

[21] W. Tunmer and K. Greaney, "Defining dyslexia," Journal of Learning Disabilities, vol. 43 pp. 229-43, 2010.

[22] M. Turner, Psycho;Ogical Assessment of Dyslexia, London: Whurr, 1997.

[23] K. Wedell, "British orientations to specific learning difficulties," in Research and Global Perspectives in Learning Disabilities: Essay in Honor of William M. Cruickshank, D. P. Hallahan and B. K. Keogh eds., Mahwah, NJ: Lawrence Erlbaum Associates, pp. 239-54, 2001.

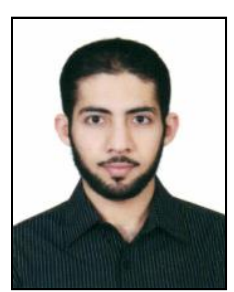

Fahad Alnaim was born in Saudi Arabia in 1985 He studied his undergraduation at King Faisal University KFU in Saudi Arabia. His major was 'special educational needs' where 'learning difficulties' was the specialism. When he graduated from King Faisal University, he was appointed there to be a teacher at the College of Education and consequentially, focused on gifted education as a specialised knowledge area; then, he was sent by the KFU to study MA gifted education in Brunel University. Accordingly, he awarded master degree with merit in 2011 . He is now a PhD student at University of Manchester searching in the area of learning disabilities LD. 